\title{
Bursting dynamics of viscous film without circular symmetry: The effect of confinement
}

\author{
Mayuko Murano and Ko Okumura* \\ Department of Physics and Soft Matter Center, Ochanomizu University, 2-1-1 Otsuka, \\ Bunkyo-ku, Tokyo 112-8610, Japan
}

(Received 11 April 2017; revised manuscript received 3 October 2017; published 13 March 2018)

\begin{abstract}
We experimentally investigate the bursting dynamics of a confined liquid film suspended in air and find a viscous dynamics distinctly different from the unconfined counterpart due to lack of circular symmetry in the shape of the expanding hole: the confined-viscous bursting proceeds at a constant speed and a rim formed at the bursting tip does not grow. We find a confined-viscous to confined-inertial crossover, as well as an unconfined-inertial to confined-inertial crossover, at which bursting speed does not change although the circular symmetry in the hole shape breaks dynamically.
\end{abstract}

DOI: 10.1103/PhysRevFluids.3.031601

\section{INTRODUCTION}

Bursting of liquid thin film is important in many fields and plays crucial roles in many industrial processes: it is observed with polymer foams [1], in glass furnaces [2], and during volcanic eruption [3]. This phenomenon is familiar to everyone as the rupture of soap films, i.e., liquid films suspended in air [4-7]. Different dynamics have been revealed for bursting of fluid film surrounded by viscous liquid (e.g., air films created in water) through studies on antibubbles [8,9], emulsions, etc. [10,11]. Dewetting, i.e., bursting of a film deposited on a substrate, is another topic that has attracted much attention [12-14]. More practically, bursting is an important tool for the generation of droplets [6,15-20] or emulsions [21] with controlled sizes because at a later stage a liquid rim formed at the bursting tip destabilizes to break into droplets. The size of droplets is a crucial factor in various fields such as environmental science [22] or disease transfer [23] because contamination results often via droplets. Bubble bursting is also important for bioreactor efficiency, because bursting is one of the causes of cell damage [24-26].

Taking a closer look at the bursting of a liquid film suspended in air, which is the basis of all the above-mentioned issues, only two scaling regimes have been established: one is called the inertial regime and the other is the viscous (or viscoelastic) regime. For almost inviscid films such as soap films or smectic films, the so-called inertial regime has been reported in which a film bursts at a constant velocity [4-7]. This velocity is known as the Taylor-Culick velocity $U_{C}=\sqrt{2 \gamma /(\rho h)}$, which results from the balance between capillarity and inertia $(\gamma, \rho$, and $h$ are the surface tension, density of the liquid, and the thickness of the film, respectively). In contrast, for ultraviscous films of polymer solutions, the viscous regime has been reported, in which bursting accelerates exponentially with time [2,27]. The characteristic time for the exponential dynamics is given by $\tau_{v} \sim \eta h / \gamma$ with $\eta$ the viscosity of the liquid. The physical origin of the viscous regime has been a contentious issue.

\footnotetext{
*okumura@phys.ocha.ac.jp
}

Published by the American Physical Society under the terms of the Creative Commons Attribution 4.0 International license. Further distribution of this work must maintain attribution to the author(s) and the published article's title, journal citation, and DOI. 
(a)

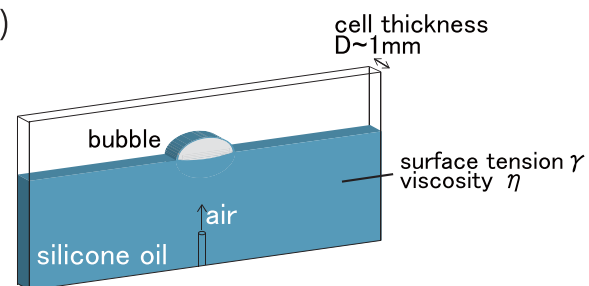

(c)

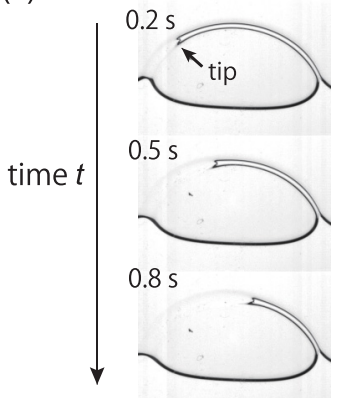

(d) $V=\frac{d r}{d t}$ : bursting velocity

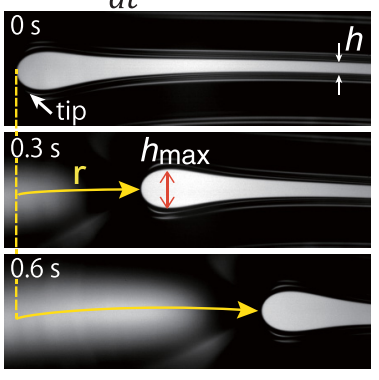

(b)

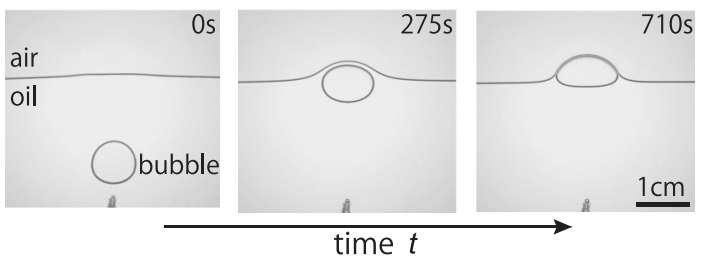

(e)

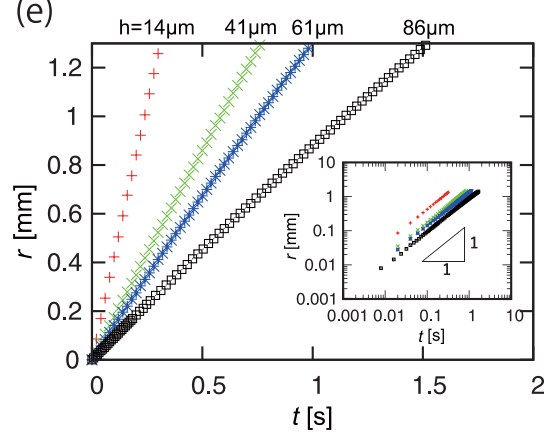

FIG. 1. (a) Experimental setup. (b) Dynamics of a bubble in the cell well before the film bursting starts ( $\eta=9.7 \mathrm{~Pa} \mathrm{~s})$. (c) Image sequence of a bubble bursting $(\eta=4.8 \mathrm{~Pa} \mathrm{~s})$. (d) Magnified snapshots of a bursting tip taken at a fixed time interval ( $\eta=9.7 \mathrm{~Pa} \mathrm{~s}$ ). See Fig. 4(a) for $h_{\max }$. (e) Bursting tip position $r$ vs time $t$ obtained from films with different thickness $h(D=0.97 \mathrm{~mm}, \eta=9.7 \mathrm{~Pa} \mathrm{~s})$. The shapes of tips in (c) and (d) look different because focal points of the photograph are different (compare SM movie 1 with 2).

Some considered that the regime is realized by the elastic feature of entangled polymers and termed the regime "viscoelastic" $[2,3,27]$, while others showed that this regime can be reproduced in a purely viscous liquid via numerical simulation and analytical theory [28-30].

While there still remains a controversy even for unconfined three-dimensional (3D) bursting, studies have been very limited for bursting of spatially confined films suspended in air despite their fundamental importance. In fact, a recent study on liquid-drop coalescence [11,31] suggests the importance of the study of confined quasi-two-dimensional (2D) bursting in air, demonstrating distinct confinement effects on the dynamics. In addition, such a confined dynamics should play fundamentally important roles in many practical applications such as those related to foams [32-34], given that any films in foam are inherently confined by Plateau borders.

Here, we study the dynamics of viscous film bursting in a confined geometry of the Hele-Shaw cell. As a result, we show by clear agreement between theory and experiment that bursting of the film sandwiched by the walls exhibits a remarkably different viscous dynamics compared with the unconfined case in three dimensions. In the unconfined case, the bursting accelerates with time without rim formation in the viscous regime (unconfined-viscous regime), and the bursting proceeds with a constant velocity with a growing rim in the inertial regime (unconfined-inertial regime). However, in the quasi-2D confined case realized by using the Hele-Shaw geometry, films burst at a constant velocity with a rim of fixed size in the viscous regime (confined-viscous regime). As for the confined-inertial dynamics, the bursting proceeds with a constant velocity, which is equal to the unconfined-inertial velocity. We experimentally confirmed the unconfined-to-confined inertial crossover with no change in bursting velocity when the circular symmetry of the shape of the expanding hole is dynamically broken.

\section{EXPERIMENT}

We fabricate a Hele-Shaw cell from two acrylic plates separated by the distance $D$ [Fig. 1(a)]. The cell thickness $D$ is adjusted by spacers and is directly measured using a laser distance sensor 
(ZS-HLDS5, Omron). We fill the cell with silicone oil $\left(\eta=0.97 \sim 48 \mathrm{~Pa} \mathrm{~s}, \rho=965 \mathrm{~kg} / \mathrm{m}^{3}, \gamma=\right.$ $20 \mathrm{mN} / \mathrm{m}$ ) and inject a bubble from the bottom of the cell. The bubble slowly rises in the viscous oil up to the liquid-air interface [Fig. 1(b)], stays there for a while, and suddenly bursts and disappears. The bursting is recorded by a high speed camera (FASTCAM SA-X, Photoron) with a macro lens (AF-S Micro NIKKOR $60 \mathrm{~mm} f / 2.8 \mathrm{GED}$, Nikon) or a microscope lens (PLN10 $\times$ or PLN4 $\times$, OLYMPUS). The point where bursting starts is not controlled and the point can be any place (e.g., places near the edges) on the liquid film encapsulating the bubble. We wait until the film bursts without any artificial trigger, which means that the thickness at the moment of bursting is not controlled because the film of the bubble keeps thinning while the bubble stays at the liquid-air interface [35]. Because of this, when the bursting starts shortly after the bubble reaches the interface, the thickness of the bursting film is relatively thick. In such a case, the bursting is found to be in a viscous regime (as shown in Sec. III). On the contrary, when the bursting starts after the film thickness becomes significantly thin, the dynamics is found to be in the inertial regime as discussed in Sec. IV.

\section{BURSTING DYNAMICS IN THE VISCOUS REGIME}

Figures 1(c)-1(e) demonstrate experimental results on the dynamics of bursting tips in the viscous regime (see Supplemental Material (SM) movie 1 [36]). Snapshots of a whole bubble and a magnified tip taken after bursting starts from the left edge are shown in Figs. 1(c) and 1(d), respectively. It is demonstrated by the three snapshots taken at a regular interval in Fig. 1(d) and by the relation between the tip position $r$ and elapsed time $t$ given in Fig. 1(e) that the bursting velocity is constant, while the velocity depends on the film thickness as shown in Fig. 1(e). Here, $r$ is the arclength between the (present) tip position and a previous one in a certain reference snapshot as indicated in Fig. 1(d). As indicated in Fig. 1(d) and verified in Sec. III A, although a rim exists at the tip, the rim does not grow as the bursting proceeds.
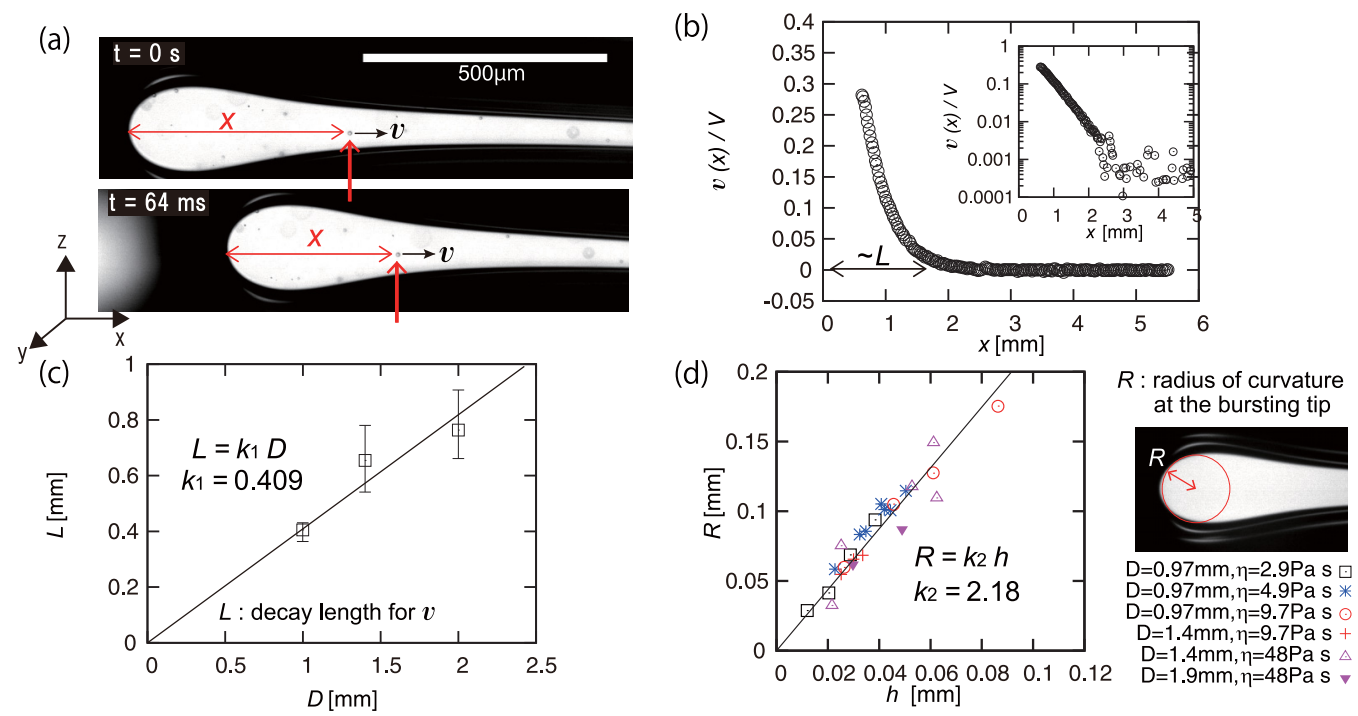

FIG. 2. (a) Example images for analysis of the flow inside the film by adding a small number of particles to oil $(D=1.0 \mathrm{~mm}, \eta=4.8 \mathrm{~Pa} \mathrm{~s}, h=44 \mu \mathrm{m})$. The thick vertical arrows indicate the horizontal position of the particle in question. (b) The velocity $v$ normalized by the bursting velocity $V$ vs distance from the tip $x(D=1.0 \mathrm{~mm}, \eta=4.8 \mathrm{~Pa} \mathrm{~s}, h=44 \mu \mathrm{m})$, obtained by the velocity change of a single particle. Inset: Semilogarithmic plot of the same data showing an exponential decay of $v$. (c) Decay length $L$ vs cell thickness $D$. Each point shows the average of four data points, and error bars indicate maximum and minimum values. The straight line shows $L=k_{1} D$ with $k_{1}=0.409 \pm 0.026$. (d) Radius of curvature at the bursting tip $R$ vs thickness $h$ on a log-log scale. The straight line shows $R=k_{2} h$ with $k_{2}=2.18 \pm 0.05$. 
As shown in Fig. 2(a), we add particles (techpolymer MBX-20, Sekisui Plastics) into oil to see the flow inside the film. The movement of particles reveals that the $y$ and $z$ components of the flow are practically zero (see SM Movie 2 [36] and Sec. IIIB) and that the remaining $x$ component is constant in the $z$ direction (see Sec. IIIC).

The length of the disturbed region $L$ increases with $D$. In Fig. 2(b), the $x$ component $v$ of flow velocity normalized by bursting velocity $V$ is given as a function of the distance from the tip $x$ on the basis of the particle-tracking analysis. As revealed in the inset of Fig. 2(b), the flow profile $v$ exhibits an exponential decay with $x$, from which we determine the decay length $L$ (see Sec. A of the SM [37] for further details). Figure 2(c) shows that $L$ increases with $D$.

As for the shape of a bursting tip, our analysis indicates that the radius of curvature at the bursting tip $R$ is determined by $h$. Figure 2(d) shows $R$, which is determined by fitting the shape of the rim to a parabolic function, as a function of the film thickness $h$ for different $D$ and $\eta$. This indicates that $R$ increases with $h$ and is independent of $D$ or $\eta$.

Theoretically, the observed bursting velocity can be explained by a global balance between the surface and dissipative energies under an assumption consistent with Fig. 2(c). The bursting is driven by a decrease in the surface energy of the film, which is dimensionally estimated as $d(\gamma D r) / d t=$ $\gamma D V$ per unit time. The viscous dissipation is estimated as follows. As discussed above, the only non-negligible component of the velocity vector is the $x$ component $(\sim V)$. The velocity gradient for this component inside the film develops in both $x$ and $y$ directions and both gradients scale as $V / D$. Note that in the $y$ direction the film is sandwiched by walls and thus the flow profile is of Poiseuille type with the velocity gradient $V / D$, and the velocity gradient in the $x$ direction $V / L$ scales as $V / D$ under the assumption $L \sim D$, which is consistent with Fig. 2(c). The velocity gradient $V / D$ originating from these two origins is developed in a volume of the order of $h D L$ with $L \sim D$. Thus, the viscous energy dissipation is described as $\eta(V / D)^{2} h D^{2}$. From the balance of the two energies, $\gamma D V \sim \eta(V / D)^{2} h D^{2}$, we obtain the bursting velocity $V=U_{\eta}$, where

$$
U_{\eta}=k \frac{\gamma}{\eta} \frac{D}{h}
$$

with a numerical coefficient $k$ to be determined experimentally. (See Sec. B of the SM [37] for another derivation of Eq. (1), which is consistent with Fig. 2(d).)

As shown in Fig. 3, Eq. (1) agrees well with experimental results. The results of measurements of $V$ as a function of $h$ for various $D$ and $\eta$ are shown in Fig. 3(a). The same data are replotted with both axes renormalized according to Eq. (1) in Fig. 3(b), in which a clear collapse of the data is demonstrated with $k$ in Eq. (1): $k=0.0350 \pm 0.0005$.

\section{A. No rim growth in the confined-viscous regime}

As shown in Fig. 4, the rim does not grow with time in the confined-viscous regime. The plot in Fig. 4(a) shows the maximum thickness $h_{\max }$ of a film as a function of the tip position $r$ [see Fig. 1(d)], which confirms that the change in the size of a rim is negligibly small. For comparison, the size of a rim in the case in which the rim grows under the volume conservation is indicated with a dotted line for $h=53 \mu \mathrm{m}$. This estimation is obtained in the following way (this is in fact an underestimate as explained below). The volume of the disappeared portion of liquid film when the bursting proceeds by the distance $r$ is $h r D$. If the liquid of this volume $h r D$ is collected into a cylindrical rim of length $D$ with the diameter $h_{\max }(r)$, we obtain $\pi\left(h_{\max }(r) / 2\right)^{2}=\pi\left(h_{\max }(r=0) / 2\right)^{2}+r h$. This yields $h_{\max }(r)=\sqrt{h_{\max }(r=0)^{2}+4 r h / \pi}$, which is used to draw the dashed line in Fig. 4(a). The present estimate is an underestimate, and the actual rim size, if the volume were conserved, would be much larger. This is because the point $r=0$ is not the position at the starting point of bursting but a position of the tip at a certain time after the initiation of bursting.

Of course, we consider that the volume is conserved as a whole: the liquid at the bursting tip is left behind on the walls and eventually drains downward along the surface of the walls (see SM Movie 2 [36]). It is a salient feature of the confined quasi-2D bursting that the fluid is not collected 

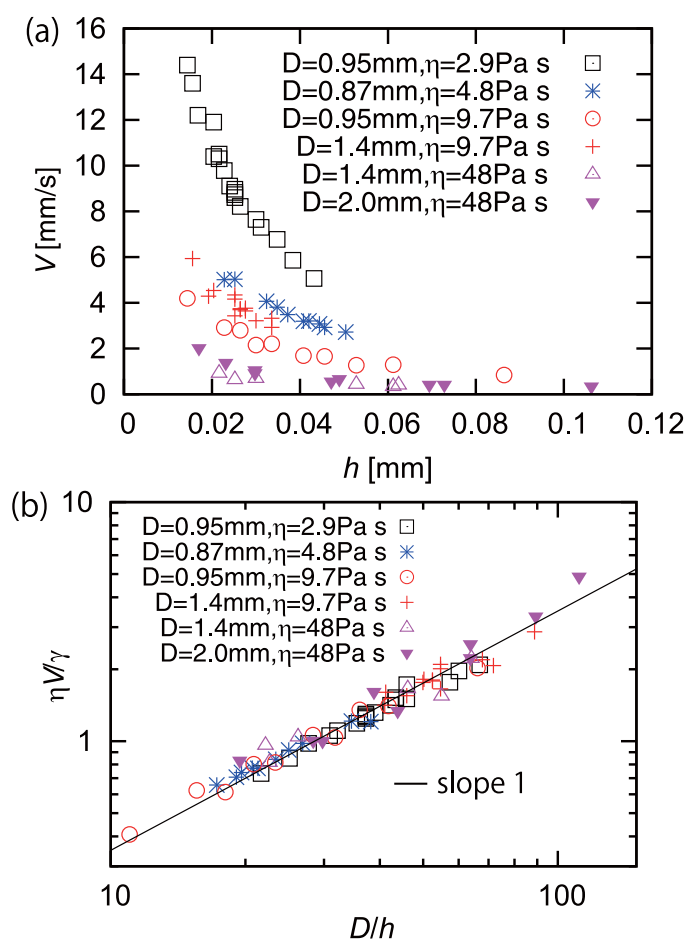

FIG. 3. (a) Bursting velocity $V$ vs film thickness $h$. (b) Normalized velocity $\eta V / \gamma$ vs normalized thickness $D / h$.

in the rim, and it never occurs in the 3D bursting in which the circular symmetry of the bursting hole is maintained. This quasi-2D feature [see Fig. 4(b)] is also reported in the study on the bursting of a liquid film sandwiched by another immiscible liquid in a Hele-Shaw cell [11].

\section{B. Absence of the $y$ and $z$ components of the flow velocity}

From the following two reasons, we conclude that the direction of flow is in the $x$ direction except in the close vicinity of the edge of the rim (bursting tip). In other words, the velocity vector has only the $x$ component with the $y$ and $z$ components being negligible.
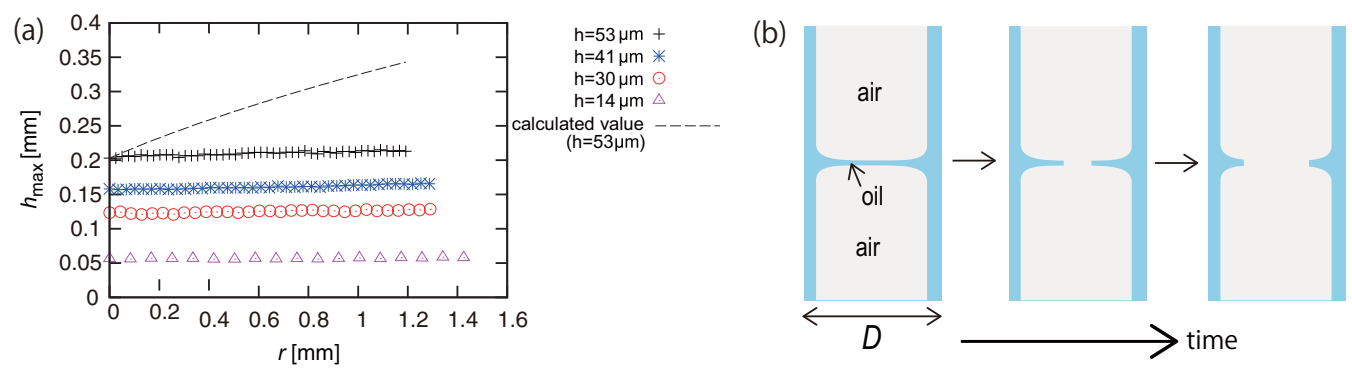

FIG. 4. (a) The maximum rim thickness in the $z$ direction during bursting $h_{\max }$ [see Fig. 1(d)] as a function of the tip position $r$ from respective reference positions for various $h(D=0.97 \mathrm{~mm}, \eta=9.7 \mathrm{~Pa} \mathrm{~s})$. The dashed line indicates the size of a rim in the case in which the rim grows under the volume conservation for $h=53 \mu \mathrm{m}$. See the text for details. (b) Side view of a film bursting. The bursting proceeds in the direction perpendicular to the paper. After the oil film bursts, the liquid "shrinks" toward the walls, and then it is absorbed into the oil films that cover the surfaces of the walls. 

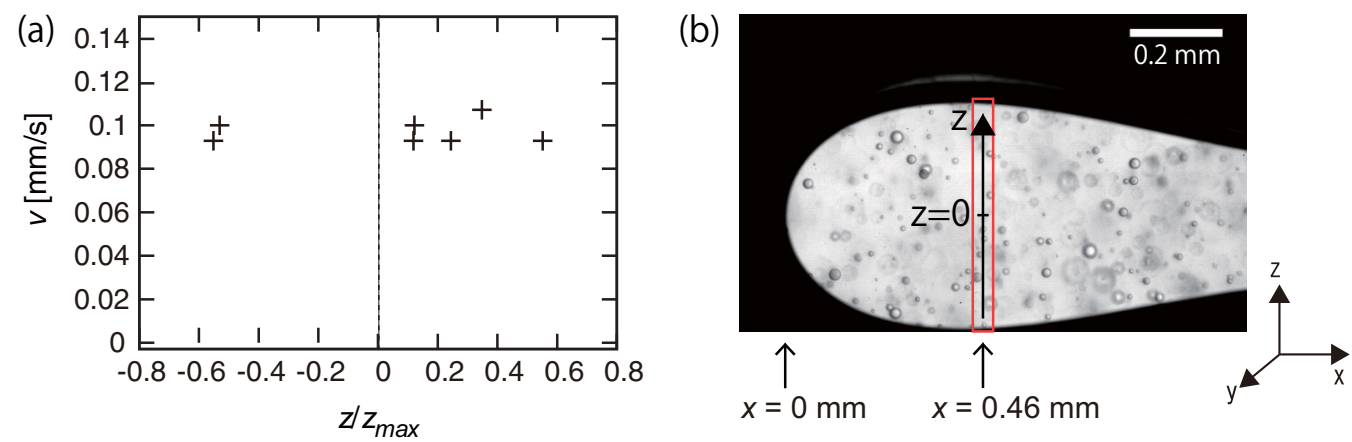

FIG. 5. The relation between the velocity in the $x$ direction and the position in the $z$ direction for particles whose $x$ coordinate of the position is in the range suggested by the narrow rectangle in (b) for $D=1.4 \mathrm{~mm}$, $\eta=4.8 \mathrm{~Pa} \mathrm{~s}$, and $h=147 \mu \mathrm{m}$. $z_{\max }$ is the position of the upper film surface at the central position of the narrow rectangle, i.e., at $x=0.46 \mathrm{~mm}\left(z_{\max }=0.24 \mathrm{~mm}\right)$, and the origin of the $z$ coordinate is placed at the center of the film.

(1) The component of flow velocity in the $y$ direction is considered to be negligibly small except in the close vicinity of the edge of the rim. The depth of the focus in the magnified movie (see SM Movie 1 [36]) is about 50-100 $\mu \mathrm{m}$. During a typical particle tracking, when a particle moves $1 \mathrm{~mm}$ in the $x$ direction, the particle stays in the range of the focal depth. This means that the $y$ component of the velocity vector is very small. Note that at positions very close to the edge of the rim, some particles disappear from view, which implies the existence of the flow in the $y$ direction in the close vicinity of the edge (see SM Sec. A [37] for further details).

(2) The component of the flow velocity in the $z$ direction is considered to be negligibly small. Even in the case of a relatively thick film $(h=147 \mu \mathrm{m})$, a particle moves only $8.6 \mu \mathrm{m}$ in the $z$ direction when it moves $1 \mathrm{~mm}$ in the $x$ direction. This implies that the flow in the $z$ direction is negligible.

Moreover, the flow inside the tip is stationary in a coordinate system fixed on the bursting tip. This is confirmed by comparing the velocity profiles obtained from different particles, which are the same within experimental errors. From these facts, we conclude that the flow field can be obtained through the movement of a single particle.

\section{Velocity gradient in the $z$ direction}

Figure 5 shows the velocity (in the $x$ direction) of different particles whose $x$ positions (from the bursting tip) are in the narrow range around the fixed value $x=0.46 \mathrm{~mm}$ from the bursting tip ( $D=1.4 \mathrm{~mm}, \eta=4.8 \mathrm{~Pa} \mathrm{~s}, h=147 \mu \mathrm{m}$ ). This plot supports that the velocities of these particles are almost the same and the velocity gradient in the $z$ direction is negligibly small. Note that as explained in Sec. IIIB, the flow velocity vector has only the $x$ component (the $y$ and $z$ components are practically zero), which is denoted as $v$ in the plot.

\section{BURSTING DYNAMICS IN THE INERTIAL REGIME}

As the Reynolds number Re increases, the confined-viscous regime exhibits a crossover to the confined-inertial regime at $\operatorname{Re} \sim 1\left(\operatorname{Re} \sim 10^{-3}\right.$ in Figs. 1-3). Here, $\operatorname{Re}$ is estimated as $\rho V D / \eta$ by considering the ratio of the inertial force $F_{i}=d(M V) / d t=\rho D h V^{2}$ (with the mass of a rim $M=\rho r D h$ ) to the viscous force $F_{v} \sim \eta V h D^{2} / D^{2}$ (see SM Sec. C for other derivations of Re, which includes an argument supporting $L \sim D$ suggested in Fig. 2(c) [37]). Note that the bursting in the confined-inertial regime is assumed to proceed at a constant speed $(V=d r / d t)$ as experimentally observed. 
(a)
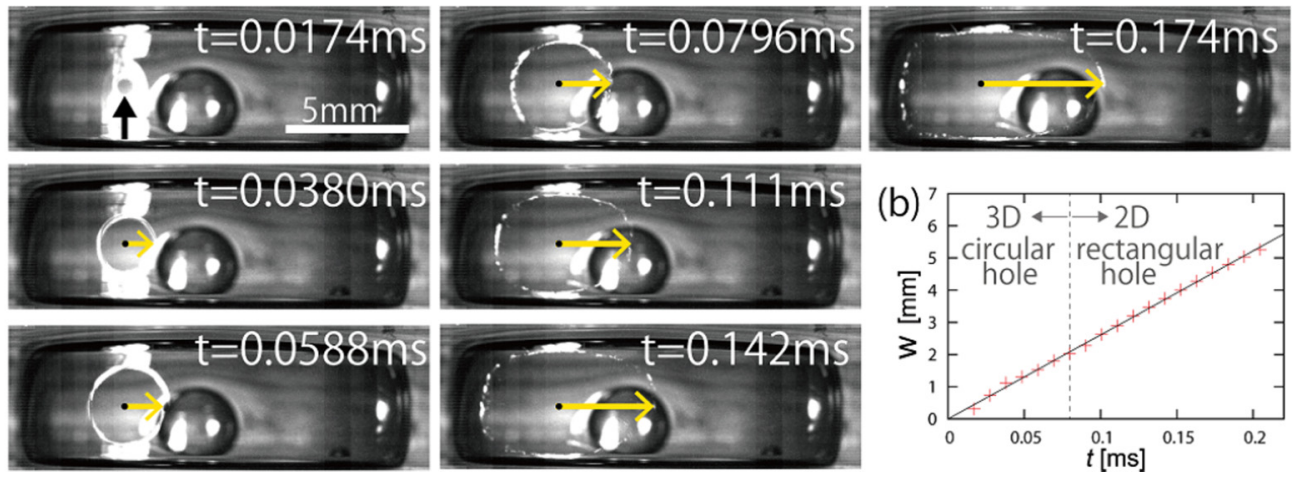

FIG. 6. (a) Sequential images of a bursting bubble taken from above ( $D=6 \mathrm{~mm}, \eta=0.0965 \mathrm{~Pa} \mathrm{~s})$. The vertical arrows in the first shot indicate the nucleation point of bursting. The horizontal yellow arrows define the half width of the hole, $w$. The small bubble observed at the center of each snapshot is irrelevant to the bursting in question. (b) Half width of the hole $w$ (indicated by horizontal arrows in snapshots) vs time $t$. The constant velocity $(\simeq 26.1 \mathrm{~m} / \mathrm{s})$ is maintained during the change in the hole shape from circular to rectangular at $t=0.0729 \mathrm{~ms}$, indicated by the dashed line. This dimensional crossover occurs before $w$ becomes $D / 2$, because films exist on the sidewalls; the hole size in the direction of cell thickness never reaches $D / 2$.

The bursting velocity $U_{i}$ in the confined-inertial regime is derived from the balance between capillary force $2 \gamma D$ and inertial force $F_{i}$, which results in $U_{i}=\sqrt{2 \gamma /(\rho h)}$. This velocity is the same with that in the unconfined $3 \mathrm{D}$ case including the numerical coefficient, i.e., $U_{i}=U_{C}$.

The confined-inertial bursting predicted above can be observed in experiment for $\operatorname{Re} \gg 1$ (see Fig. 6, in which $\operatorname{Re} \sim 20$ ). In Fig. 6(a), the expanding hole grows at first maintaining the circular shape as in the case of the unconfined 3D film and then changes its shape to a quasirectangular one, but the bursting speed is unchanged throughout bursting as shown in Fig. 6(b). This observation is consistent with the above prediction in the following manner. First, the observed initial 3D bursting is in the inertial regime because, if a bursting proceeds at a constant speed for a 3D film, the bursting velocity is $U_{C}$, as we mentioned in Sec. I. Second, since Re $\gg 1$ is satisfied, the observed rectangular bursting is expected to be in the confined-inertial regime and thus proceeds with the velocity $U_{i}$, which is observed to be equal to the unconfined-inertial velocity $U_{C}$, as predicted. Finally, the rim growth, which we suppose in the deviation of $U_{i}$, is consistent with experimental observation. This is because, at the bursting tip, drop generation can be seen, which is caused by fragmentation of an amply grown rim into small droplets (see SM Movie 3 [36]).

\section{DISCUSSION}

At very short times after the nucleation of an initial hole for bursting before the confined-viscous regime sets in, the film seems to rupture at a velocity dozens times higher than $U_{\eta}$. If we could capture this initial stage of bursting, we would see the change in the shape of the expanding hole from circular (unconfined 3D) to quasirectangular (confined quasi-2D) in the viscous regime as observed in the inertial bursting. However, this ultrafast regime is difficult to capture and requires a separate study. This is because the control of the point where bursting starts is technically difficult and the time scales for the ultrafast initial regime and the viscous regime are extremely different.

In Ref. [38], the authors confined a film between two needles and punctured the film with another needle to measure the bursting velocity and reported a bursting velocity different from ours. This difference may originate from the difference between the needle and Hele-Shaw geometries and/or a significant difference in characteristic length scales of the two experiments.

The present work could make a significant contribution to the field of bursting films in air, given that only a few scaling regimes have been known despite the long history of research. For 
example, this work provides fundamentally important knowledge for understanding the dynamics of foams in general [34]. Controlling the rim growth with the aid of Eq. (1) may be useful for environmental problems or industrial applications associated with the generation of droplets $[22,23,39]$. Furthermore, Eq. (1) and the experimentally confirmed invariance of the bursting speed $\left(U_{i}=U_{C}\right)$ at the $3 \mathrm{D}$ to quasi-2D crossover in the inertial regime could be useful for measuring the thickness of confined liquid films in a wide range from micron to nanometer scales.

\section{ACKNOWLEDGMENTS}

The authors thank Natsuki Kimoto for making trials for the initiation of this research project. This research was partly supported by a Grant-in-Aid for Scientific Research (A) (Grant No. 24244066) of the Japan Society for the Promotion of Science (JSPS), Japan, and by the ImPACT Program of the Council for Science, Technology and Innovation (Cabinet Office, government of Japan) (Grant No. 2014-PM01-02-01). M.M. is supported by a JSPS Science Research Fellowship for Young Scientists (Grant No. 16J00871).

[1] L. J. Lee, C. Zeng, X. Cao, X. Han, J. Shen, and G. Xu, Polymer nanocomposite foams, Compos. Sci. Technol. 65, 2344 (2005).

[2] G. Debrégeas, P.-G. De Gennes, and F. Brochard-Wyart, The life and death of "bare" viscous bubbles, Science 279, 1704 (1998).

[3] P.-G. De Gennes, F. Brochard-Wyart, and D. Quéré, Capillarity and Wetting Phenomena: Drops, Bubbles, Pearls, Waves (Springer, New York, 2013).

[4] G. Taylor, The dynamics of thin sheets of fluid. III. Disintegration of fluid sheets, Proc. R. Soc. London A 253, 313 (1959).

[5] W. R. McEntee and K. J. Mysels, Bursting of soap films. I. An experimental study, J. Phys. Chem. 73, 3018 (1969).

[6] A. B. Pandit and J. F. Davidson, Hydrodynamics of the rupture of thin liquid films, J. Fluid Mech. 212, 11 (1990).

[7] F. Müller, U. Kornek, and R. Stannarius, Experimental study of the bursting of inviscid bubbles, Phys. Rev. E 75, 065302 (2007).

[8] S. T. Thoroddsen, M. J. Thoraval, K. Takehara, and T. G. Etoh, Micro-bubble morphologies following drop impacts onto a pool surface, J. Fluid Mech. 708, 469 (2012).

[9] J. Zou, C. Ji, B. G. Yuan, X. D. Ruan, and X. Fu, Collapse of an antibubble, Phys. Rev. E 87, 061002 (2013).

[10] É. Reyssat and D. Quéré, Bursting of a fluid film in a viscous environment, Europhys. Lett. 76, 236 (2006).

[11] A. Eri and K. Okumura, Bursting of a thin film in a confined geometry: Rimless and constant-velocity dewetting, Phys. Rev. E 82, 030601(R) (2010).

[12] C. Redon, F. Brochard-Wyart, and F. Rondelez, Dynamics of Dewetting, Phys. Rev. Lett. 66, 715 (1991).

[13] G. Reiter, M. Hamieh, P. Damman, S. Sclavons, G. Sylvain, T. Vilmin, and E. Raphäl, Residual stresses in thin polymer films cause rupture and dominate early stages of dewetting, Nat. Mater. 4, 754 (2005).

[14] P. Damman, S. Gabriele, S. Coppée, S. Desprez, D. Villers, T. Vilmin, E. Raphaël, M. Hamieh, S. Al Akhrass, and G. Reiter, Relaxation of Residual Stress and Reentanglement of Polymers in Spin-Coated Films, Phys. Rev. Lett. 99, 036101 (2007).

[15] D. E. Spiel, On the births of film drops from bubbles bursting on seawater surfaces, J. Geophys. Res. 103, 24907 (1998).

[16] H. Lhuissier and E. Villermaux, Soap Films Burst Like Flapping Flags, Phys. Rev. Lett. 103, 054501 (2009).

[17] J. C. Bird, R. D. Ruiter, L. Courbin, and H. A. Stone, Daughter bubble cascades produced by folding of ruptured thin films, Nature (London) 465, 759 (2010).

[18] P. L. L. Walls, L. Henaux, and J. C. Bird, Jet drops from bursting bubbles: How gravity and viscosity couple to inhibit droplet production, Phys. Rev. E 92, 021002 (2015). 
[19] B. Y. Ni, A. M. Zhang, and G. X. Wu, Simulation of a fully submerged bubble bursting through a free surface, Eur. J. Mech. B Fluid 55, 1 (2016).

[20] C. Vernay, L. Ramos, and C Ligoure, Bursting of Dilute Emulsion-Based Liquid Sheets Driven by a Marangoni Effect, Phys. Rev. Lett. 115, 198302 (2015).

[21] J. Feng, M. Roché, D. Vigolo, L.N. Arnaudov, S. D. Stoyanov, T. D. Gurkov, G. G. Tsutsumanova, and H. A. Stone, Nanoemulsions obtained via bubble-bursting at a compound interface, Nat. Phys. 10, 606 (2014).

[22] J. Wu, Evidence of sea spray produced by bursting bubbles, Science 212, 324 (1981).

[23] L. T. Angenent, S. T. Kelley, A. S. Amand, N. R. Pace, and M. T. Hernandez, Molecular identification of potential pathogens in water and air of a hospital therapy pool, Proc. Natl. Acad. Sci. USA 102, 4860 (2005).

[24] R. S. Cherry and C. T. Hulle, Cell death in the thin films of bursting bubbles, Biotechnol. Progr. 8, 11 (1992).

[25] W. Hu, C. Berdugo, and J. J. Chalmers, The potential of hydrodynamic damage to animal cells of industrial relevance: Current understanding, Cytotechnology 63, 445 (2011).

[26] Y. Liu, F. Li, W. Hu, K. Wiltberger, and T. Ryll, Effects of bubble-liquid two-phase turbulent hydrodynamics on cell damage in sparged bioreactor, Biotechnol. Progr. 30, 48 (2014).

[27] G. Debrégeas, P. Martin, and F. Brochard-Wyart, Viscous Bursting of Suspended Films, Phys. Rev. Lett. 75, 3886 (1995).

[28] M. P. Brenner and D. Gueyffier, On the bursting of viscous films, Phys. Fluids 11, 737 (1999).

[29] G. Sünderhauf, H. Raszillier, and F. Durst, The retraction of the edge of a planar liquid sheet, Phys. Fluids 14, 198 (2002).

[30] N. Savva and J. W. M. Bush, Viscous sheet retraction, J. Fluid Mech. 626, 211 (2009).

[31] K. Okumura, Adv. Colloid Interface Sci. (2017), doi:10.1016/j.cis.2017.07.021.

[32] H. Ritacco, F. Kiefer, and D. Langevin, Lifetime of Bubble Rafts: Cooperativity and Avalanches, Phys. Rev. Lett. 98, 244501 (2007).

[33] A-L. Biance, A. Delbos, and O. Pitois, How Topological Rearrangements and Liquid Fraction Control Liquid Foam Stability, Phys. Rev. Lett. 106, 068301 (2011).

[34] I. Cantat, S. Cohen-Addad, F. Elias, F. Graner, R. Höhler, O. Pitois, F. Rouyer, and A. Saint-Jalmes, Les mousses-Structure et dynamique (Éditions Belin, Paris, 2010).

[35] A. Eri and K. Okumura, Lifetime of a two-dimensional air bubble, Phys. Rev. E 76, 060601 (2007).

[36] See Supplemental Material at http://link.aps.org/supplemental/10.1103/PhysRevFluids.3.031601 for movies of bursting in the viscous regime (Movies 1 and 2) and inertial regime (Movie 3).

[37] See Supplemental Material at http://link.aps.org/supplemental/10.1103/PhysRevFluids.3.031601 for further text descriptions.

[38] S. V. Chepushtanova and I. L. Kliakhandler, Slow rupture of viscous films between parallel needles, J. Fluid Mech. 573, 297 (2007).

[39] A. Gupta, H. B. Eral, T. A. Hatton, and P. S. Doyle, Nanoemulsions: Formation, properties and applications, Soft Matter 12, 2826 (2016). 